\title{
A COMPARISON BETWEEN MICROSOFT EXCEL SOLVER AND NCSS, SPSS ROUTINES FOR NONLINEAR REGRESSION MODELS
}

\author{
Didem Tetik Küçükelçia ${ }^{a *}$ Atıf Evren ${ }^{b}$ \\ $a^{*}$ Department of Statistics, Faculty of life and natural science, University of Yildiz Technical \\ University Istanbul, Turkey \\ didemkucukelci@yahoo.com.tr (corresponding author) \\ ${ }^{b}$ Department of Statistics Faculty of life and natural science, \\ Yildiz Technic University Istanbul, Turkey \\ aevren@yildiz.edu.tr
}

\begin{abstract}
The aim of this paper is by comparing the results obtained by Microsoft Excel Solver programme with those of NCSS and SPSS in some nonlinear regression models. We fit some nonlinear models to data present in http//itl.nist.gov/div898/strd/nls/nls_main.shtml by the three packages. Excel did succeed enough; we conclude that it provides us a cheaper and a more interactive way of studing nonlinear models.
\end{abstract}

Keywords: Non-Linear Regression, SPSS, NCSS, EXCEL solver.

\section{Nonlinear regression model}

Nonlinear regression models use the same form with linear regression models with some exceptions. First of all, an observation on the dependent variable can be seen as a sum of a mean response estimated by a nonlinear function and generally an additive random error term(1). Secondly, the analytic solutions of the normal equations for nonlinear models are very difficult to reach. Therefore some iterative numerical search procedures are required. Thirdly, parameter estimates are to be obtained iteratively such that different initial estimates may yield totally different final estimates. Fourthly, in nonlinear regression models, there may be more than one canditate model that fit data simultaneously. Finally, inferences about nonlinear regression parameters are usually based on large-sample theory which means that the validities of hypothesis testing and interval estimation procedures depend mostly on how large the samples 
are. In other words, in nonlinear models, the validity of statistical inference depends on "asymptotic normality"(1).

A nonlinear regression model can be specified as

$$
Y_{i}=f\left(X_{i j}, \gamma\right)+\varepsilon_{i}
$$

Here $Y_{i}$ is the ith observed value of dependent variable $f\left(X_{i j}, \gamma\right)$ is the nonlinear function of parameters $\gamma_{0}, \gamma_{1}, \ldots, \gamma_{p-1} . X_{i j}$ is the ith observed value of the $\mathrm{jth}$ independent variable $(j=1,2, \ldots, q)$. The matrix of the observations of independent variable is

$$
X_{q n}=\left[\begin{array}{cccc}
X_{11} X_{12} & \ldots & X_{1 n} \\
X_{21} X_{22} & \ldots & X_{2 n} \\
\ldots & \\
X_{q 1} X_{q 2} & \ldots & X_{q n}
\end{array}\right]
$$

We suppose that the model has has p parameters and the parameter vector is denoted by $\gamma$

$$
\gamma=\left[\begin{array}{c}
\gamma_{0} \\
\gamma_{1} \\
\cdots \\
\gamma_{p-1}
\end{array}\right]
$$

The vector of initial estimates for $\mathrm{p}$ parameters is $g$ as given below :

$$
g=\left[\begin{array}{c}
g_{0} \\
g_{1} \\
\ldots \\
g_{p-1}
\end{array}\right]
$$

Besides the difference between the kth parameter $(\mathrm{k}=0,1,2, \ldots, \mathrm{p}-1)$ and its initial estimate before the first iteration is realized is

$$
\beta_{k}^{(0)}=\gamma_{k}-g_{k}^{(0)}
$$

If the matrix whose entries are the first derivative values of the expectation function with respect to kth parameter at the initial estimate level is denoted by

$$
D_{i k}^{(0)}=\left[\frac{\partial f\left(X_{i}, \gamma\right)}{\partial \gamma_{k}}\right]_{\gamma=g^{(0)}}
$$

Then by Taylor expansion one can get the following linearized form

$$
Y_{i} \cong f_{i}^{(0)}+\sum_{k=0}^{p-1} D_{i k}^{(0)} \beta_{k}^{(0)}+\varepsilon_{i}
$$

And if the ith residual is defined as

$$
Y_{i}^{(0)}=Y_{i}-f_{i}^{(0)}
$$


Then another version of (1) is obtained as follows:

$$
Y_{i}^{(0)} \cong f_{i}^{(0)}+\sum_{k=0}^{p-1} D_{i k}^{(0)} \beta_{k}^{(0)}+\varepsilon_{i}
$$

Or by using matrix notation;

$$
Y^{(0)} \cong D^{(0)} \beta^{(0)}+\varepsilon
$$

This mathematical form is the same as the one used in linear models. Here the derivative matrix D plays the same role of X matrix in linear regression models. Hence parameter estimates and hypothesis tests can be realized by the help of this analogy [7].

Following this argumentation, estimators of $\beta$ can be realized similarly by the following equation:

$$
b^{(0)}=\left(D^{(0)^{T}} D^{(0)}\right)^{-1} D^{(0)^{T}} Y^{(0)}
$$

Yet, some differences should be emphasized. In nonlinear regression models, the residuals (obtained at the end of each iteration) play the same role as the observed values of explained variable in linear regression models. In other words, in linear regression models, observed values of dependent variable are being projected to two orthogonal subspaces of $\mathrm{n}$ dimensional Euclidean space to obtain residuals and parameter estimates. In nonlinear models, however, residuals themselves are projected to get parameter estimates iteratively.

At the end of each iteration current parameter estimate vector is modified by the following equation:

$$
g_{k}^{(j)}=g_{k}^{(j-1)}+b_{k}^{(j-1)} \quad \text { for } \mathrm{j}=1,2, \ldots
$$

The iterative search procedure is finished as soon as iterative parameter estimates ( or summarizing statistics like sum of squares, etc.) converge to some numbers (or the differences between iterative estimates become negligible). But this means that what one can obtain from these estimation process will probably correspond to a stationary point therefore the point reached at the end of iterations may coincide with a local optimum rather than a global one.

If we assume that the error terms are independently and normally distributed variables with zero expectation and the common variance $\sigma^{2}$ then the asymptotic sampling distribution of $\mathrm{g}$ can be approximated by a multivariate normal distribution with the expectation vector given in (13) and the estimated variance-covariance matrix in (14):

$$
\begin{gathered}
E(g) \cong \gamma \\
S^{2}(g)=\operatorname{MSE}\left(D^{T} D\right)^{-1}
\end{gathered}
$$

Here MSE is the mean squares for the error term as usual 


$$
M S E=\frac{\sum_{i=1}^{n} e_{i}^{2}}{n-p}
$$

The validity of parameter estimates realized by(10) and (11) depends on how justifiable the linearization technique is. In other words, if the intrinsic nonlinearity is high, then the results derived from the analysis of residuals may be highly misleading [9]. It is also a fact that, in practice a lot of competing algorithms are being used to solve some problems so that (10) and (11) are useful in a pedagogical way. The formulas in (13) and (14) are used in hypothesis testing and confidence interval estimation procedures . For studying bootstrap estimates in nonlinear modeling one can refer to [5].

Fitting nonlinear functions to data sometimes seems rather to be an art. To use any method, we must first determine starting values, step sizes. Neither step sizes nor starting values are necessary in linear fitting [2]. Some problems that has to be taken into account, in nonlinear regression models and some practical ways to remedy can be found in [6] and [8]. Some of these issues can be listed as follows:

i) For nonlinear models, the objective function (the least squares or the maximum likelihood function, etc.) may probably have more than one optimum (may have none for some instances)!

ii) Some of the goodness of fit statistics like R-square may be highly misleading for nonlinear models. (For nonlinear models R-square values should be very close to 1 for a good fit. In other words, the linear and nonlinear models to model the same data cannot be compared by only comparing R-square statistics [6].

iii) For nonlinear models, initial parameter estimates should be introduced iteration process exclusively. Different intial parameter estimates may yield different final estimates.

iv) In nonlinear regression models parameter estimates are not simply the linear functions of observed values of dependent variable such that the assumption on the normality of dependent variables does not guarentee for parameter estimates to distribute normally. On the other hand confidence enterval estimates heavily depend on the assumption of asymptotic normality which in turn requires bigger sample sizes.

v) Analysis of residuals in nonlinear modeling are realized in analogy with linear models which is a probable source for error. Because if intrinsic curvature is high, the results obtained by the analysis of residuals may be misleading . 


\subsection{Application}

The names of data set, and the models that we tried to fit are given in the following table:

Table 1. Models

\begin{tabular}{|c|c|}
\hline $\begin{array}{l}\text { DATASET } \\
\text { NAME }\end{array}$ & $\begin{array}{l}\text { MATHEMATICAL EXPRESSION OF THE } \\
\text { MODEL THAT FITTED TO DATA }\end{array}$ \\
\hline 1-Misra1a & $\mathrm{y}=\mathrm{b} 1 *(1-\exp [-\mathrm{b} 2 * x])+\mathrm{e}$ \\
\hline 2-Chwirut2 & $\mathrm{y}=\exp (-\mathrm{b} 1 * x) /(\mathrm{b} 2+\mathrm{b} 3 * x)+\mathrm{e}$ \\
\hline 3-Chwirut1 & $\mathrm{y}=\exp [-\mathrm{b} 1 * x] /(\mathrm{b} 2+\mathrm{b} 3 * x)+\mathrm{e}$ \\
\hline 4-Lanczos3 & $\begin{array}{l}y=b 1 * \exp (-b 2 * x)+b 3 * \exp (-b 4 * x)+b 5 * \exp (-b 6 * x) \\
+e\end{array}$ \\
\hline 5-Gauss1 & $\begin{array}{l}\mathrm{y}=\mathrm{b} 1 * \exp (-\mathrm{b} 2 * \mathrm{x})+\mathrm{b} 3 * \exp (-(\mathrm{x}-\mathrm{b} 4) * * 2 / \mathrm{b} 5 * * 2) \\
+\mathrm{b} 6 * \exp (-(\mathrm{x}-\mathrm{b} 7) * * 2 / \mathrm{b} 8 * * 2)+\mathrm{e}\end{array}$ \\
\hline 6-Gauss2 & $\begin{array}{l}\mathrm{y}=\mathrm{b} 1 * \exp (-\mathrm{b} 2 * \mathrm{x})+\mathrm{b} 3 * \exp (-(\mathrm{x}-\mathrm{b} 4) * * 2 / \mathrm{b} 5 * * 2) \\
+\mathrm{b} 6 * \exp (-(\mathrm{x}-\mathrm{b} 7) * * 2 / \mathrm{b} 8 * * 2)+\mathrm{e}\end{array}$ \\
\hline 7-Danwood & $\mathrm{y}=\mathrm{b} 1 * \mathrm{x} * \mathrm{~b} 2+\mathrm{e}$ \\
\hline 8-Misra1b & $\mathrm{y}=\mathrm{b} 1 *(1-(1+\mathrm{b} 2 * \mathrm{x} / 2) * *(-2))+\mathrm{e}$ \\
\hline 9-Kirby2 & $\begin{array}{l}\mathrm{y}=(\mathrm{b} 1+\mathrm{b} 2 * \mathrm{x}+\mathrm{b} 3 * \mathrm{x} * 2) /(1+\mathrm{b} 4 * \mathrm{x}+\mathrm{b} 5 * \mathrm{x} * * 2)+ \\
\mathrm{e}\end{array}$ \\
\hline 10-Hahn1 & $\begin{array}{l}\mathrm{y}=(\mathrm{b} 1+\mathrm{b} 2 * \mathrm{x}+\mathrm{b} 3 * \mathrm{x} * * 2+\mathrm{b} 4 * \mathrm{x} * * 3) / \\
(1+\mathrm{b} 5 * \mathrm{x}+\mathrm{b} 6 * \mathrm{x} * 2+\mathrm{b} 7 * \mathrm{x} * * 3)+\mathrm{e}\end{array}$ \\
\hline 11-Mgh17 & $y=b 1+b 2 * \exp [-x * b 4]+b 3 * \exp [-x * b 5]+e$ \\
\hline 12Lanczos1,2 & $\begin{array}{l}y=b 1 * \exp (-b 2 * x)+b 3 * \exp (-b 4 * x)+b 5 * \exp (-b 6 * x) \\
+e\end{array}$ \\
\hline 13-Gauss3 & $\begin{array}{l}\mathrm{y}=\mathrm{b} 1 * \exp (-\mathrm{b} 2 * \mathrm{x})+\mathrm{b} 3 * \exp (-(\mathrm{x}-\mathrm{b} 4) * * 2 / \mathrm{b} 5 * * 2) \\
+\mathrm{b} 6 * \exp (-(\mathrm{x}-\mathrm{b} 7) * * 2 / \mathrm{b} 8 * * 2)+\mathrm{e}\end{array}$ \\
\hline 14-Misra1c & $\begin{array}{l}\mathrm{y}=\mathrm{b} 1 * \exp (-\mathrm{b} 2 * \mathrm{x})+\mathrm{b} 3 * \exp (-(\mathrm{x}-\mathrm{b} 4) * * 2 / \mathrm{b} 5 * * 2) \\
+\mathrm{b} 6 * \exp (-(\mathrm{x}-\mathrm{b} 7) * * 2 / \mathrm{b} 8 * * 2)+\mathrm{e}\end{array}$ \\
\hline 15-Misra1d & $\mathrm{y}=\mathrm{b} 1 *(1-(1+2 * \mathrm{~b} 2 * \mathrm{x}) * *(-.5))+\mathrm{e}$ \\
\hline 16-Roszman1 & $\mathrm{y}=\mathrm{b} 1 * \mathrm{~b} 2 * \mathrm{x} *((1+\mathrm{b} 2 * \mathrm{x}) * *(-1))+\mathrm{e}$ \\
\hline 17-Enso & $\mathrm{y}=\mathrm{b} 1-\mathrm{b} 2 * \mathrm{x}-\arctan [\mathrm{b} 3 /(\mathrm{x}-\mathrm{b} 4)] / \mathrm{pi}+\mathrm{e}$ \\
\hline 18-Mgh09 & $\begin{array}{l}\mathrm{y}=\mathrm{b} 1+\mathrm{b} 2 * \cos \left(2 * \mathrm{pi}^{*} \mathrm{x} / 12\right)+\mathrm{b} 3 * \sin \left(2 * \mathrm{pi}^{*} \mathrm{x} / 12\right)+ \\
\mathrm{b} 5 * \cos (2 * \mathrm{pi} \mathrm{x} / \mathrm{b} 4)+\mathrm{b} 6 * \sin \left(2 * \mathrm{pi}{ }^{*} \mathrm{x} / \mathrm{b} 4\right)+\mathrm{b} 8 * \cos ( \\
2 * \mathrm{pi} \mathrm{x} / \mathrm{b} 7)+\mathrm{b} 9 * \sin (2 * \mathrm{pi} \mathrm{x} / \mathrm{b} 7)+\mathrm{e}\end{array}$ \\
\hline 19-Thurber & $\mathrm{y}=\mathrm{b} 1 *(\mathrm{x} * 2+\mathrm{x} * \mathrm{~b} 2) /(\mathrm{x} * 2+\mathrm{x} * \mathrm{~b} 3+\mathrm{b} 4)+\mathrm{e}$ \\
\hline 20-BoxBod & $\begin{array}{l}\mathrm{y}=(\mathrm{b} 1+\mathrm{b} 2 * \mathrm{x}+\mathrm{b} 3 * \mathrm{x} * * 2+\mathrm{b} 4 * \mathrm{x} * * 3) / \\
(1+\mathrm{b} 5 * \mathrm{x}+\mathrm{b} 6 * \mathrm{x} * * 2+\mathrm{b} 7 * \mathrm{x} * * 3)+\mathrm{e}\end{array}$ \\
\hline 21-Rat42 & $\mathrm{y}=\mathrm{b} 1 *(1-\exp [-\mathrm{b} 2 * x])+\mathrm{e}$ \\
\hline 22-Mgh10 & $\mathrm{y}=\mathrm{b} 1 /(1+\exp [\mathrm{b} 2-\mathrm{b} 3 * x])+\mathrm{e}$ \\
\hline 23-Eckerle4 & $\mathrm{y}=\mathrm{b} 1 * \exp [\mathrm{b} 2 /(\mathrm{x}+\mathrm{b} 3)]+\mathrm{e}$ \\
\hline 24-Rat43 & $\mathrm{y}=(\mathrm{b} 1 / \mathrm{b} 2) * \exp \left[-0.5^{*}((\mathrm{x}-\mathrm{b} 3) / \mathrm{b} 2)^{* * 2}\right]+\mathrm{e}$ \\
\hline 25-Bennett5 & $\mathrm{y}=\mathrm{b} 1 /((1+\exp [\mathrm{b} 2-\mathrm{b} 3 * \mathrm{x}]) * *(1 / \mathrm{b} 4))+\mathrm{e}$ \\
\hline 26-Nelson & $\log [y]=b 1-b 2 * x 1 * \exp [-b 3 * x 2]+e$ \\
\hline
\end{tabular}


In terms of R-square statistic what we have got after fitting all these models can be summarized as below:

Table 2. R-square statistics

\begin{tabular}{|c|c|c|c|}
\hline Model & $\operatorname{Excel}\left(R^{\wedge} 2\right)$ & ${\operatorname{NCSS}\left(R^{\wedge} 2\right)}$ & 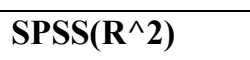 \\
\hline 1-Misra1a & 0,999 & 0,999 & 0,991 \\
\hline 2-Chwirut2 & $0,76(*)$ & 0,986 & 0,932 \\
\hline 3-Chwirut1 & $0,76(*)$ & 0,98 & 0,98 \\
\hline 4-Lanczos3 & 0,423 & 0,422 & $\begin{array}{l}\text { Convergence } \\
\text { criterion was not } \\
\text { met. }\end{array}$ \\
\hline 5-Gauss1 & $\begin{array}{l}\text { Convergence } \\
\text { criterion was } \\
\text { not met. }\end{array}$ & 0,996 & 0,997 \\
\hline 6-Gauss2 & $\begin{array}{l}\text { Convergence } \\
\text { criterion was } \\
\text { not met. }\end{array}$ & 0,996 & 0,996 \\
\hline 7-Danwood & 0,999 & 0,999 & 0,999 \\
\hline 8-Misra1b & 0,999 & 0,999 & 1 \\
\hline 9-Kirby2 & 0,99 & 0,999 & 1 \\
\hline 10-Hahn1 & 0,993 & 0,994 & 1 \\
\hline 11-Mgh17 & 0,358 & 0,359 & 0,359 \\
\hline 12-Lanczos & $\begin{array}{l}\text { Convergence } \\
\text { criterion was } \\
\text { not met.. }\end{array}$ & 0,037 -overflow & $\begin{array}{l}\text { Convergence } \\
\text { criterion was not } \\
\text { met. }\end{array}$ \\
\hline 13-Gauss3 & 0,338 & 0,996 & 0,996 \\
\hline 14-Misra1c & 0,999 & 0,999 & 1 \\
\hline 15-Misra1d & 0,999 & 0,999 & 1 \\
\hline 16-Roszman1 & 0,997 & 0,998 & 0,998 \\
\hline 17-Enso & 0,598 & 0,596 & 0,597 \\
\hline 18-Mgh09 & 0,319 & 0,705 & 0,293 \\
\hline 19-Thurber & 0,999 & 0,999 & 0,999 \\
\hline 20-BoxBod & 0,495 & 0,88 & 0,88 \\
\hline 21-Rat42 & 0,998 & 0,978 & 0,978 \\
\hline 22-Mgh10 & 0,074 & 0,006 & 0,002 \\
\hline 23-Eckerle4 & 0,997 & 0,997 & 0,997 \\
\hline 24-Rat43 & 0,991 & 0,992 & 0,992 \\
\hline 25-Bennett5 & 0,999 & 0,999 & 1 \\
\hline 26-Nelson & 0,83 & 0,79 & $\begin{array}{l}\text { Convergence } \\
\text { criterion was not } \\
\text { met. }\end{array}$ \\
\hline
\end{tabular}

\section{Some comments}

1-For the totality of 26 data sets EXCEL Solver has found 13a suitable solutions out of 26 cases $(50 \%)$ whereas NCSS has found 19 suitable solutions out of 26 cases $(73 \%)$ and SPSS has found 19 suitable solutions out of 26 cases $(73 \%)$.

2-For the cases labeled by 4,11,12,18,23 and 26; none of the programs have found a suitable solution. 
3-If the models are grouped under the titles "easy", "medium" and "puzzle" what the models have yielded is as follows:

a) For the "easy" category ( the ones from 1 to 8); EXCEL has found 3 suitable solutions out of 8 . (37\%) while NCSS and SPSS have found 7 suitable solutions out of $8(87 \%)$.

b) For the "medium" category ( the ones from 9 to 18); EXCEL succeded in 5 out of 10 situations $(50 \%)$ while NCCS and SPSS have succeeded in 6 out of 8 categories $(75 \%)$

c) For the "puzzle" category (the ones from 19 to 26 ) EXCEL has managed well 5 out of 8 situations $(62.5 \%)$ while NCSS and SPSS have done well 6 out of 8 situations $(75 \%)$.

Table 3. Final Estimates

\begin{tabular}{|c|c|c|c|c|c|}
\hline & & & & OUTPUT & \\
\hline & $\begin{array}{l}\text { DATASET } \\
\text { NAME }\end{array}$ & INPUTS & NCSS & SPSS & EXCEL \\
\hline $1-$ & Misrala & $\begin{array}{l}\mathrm{B} 1=500 \\
\mathrm{~B} 2=0.0001\end{array}$ & $\begin{array}{l}\mathrm{B} 1=1.57 . \mathrm{E} 7 \\
\mathrm{~B} 2=5.7 . \mathrm{E}-7\end{array}$ & $\begin{array}{l}\mathrm{B} 1=2.52 . \mathrm{E}-5 \\
\mathrm{~B} 2=3.5 . \mathrm{E}-5\end{array}$ & $\begin{array}{l}\mathrm{B} 1=244.6 \\
\mathrm{~B} 2=5 . \mathrm{E}-4\end{array}$ \\
\hline $2-$ & Chwirut2 & $\begin{array}{l}\mathrm{B} 1=0.1 \\
\mathrm{~B} 2=0.001 \\
\mathrm{~B} 3=0.002\end{array}$ & $\begin{array}{l}\mathrm{B} 1=0.166 \\
\mathrm{~B} 2=0.005 \\
\mathrm{~B} 3=0.012\end{array}$ & $\begin{array}{l}\mathrm{B} 1=0.077 \\
\mathrm{~B} 2=0.003 \\
\mathrm{~B} 3=0.017\end{array}$ & $\begin{array}{l}\mathrm{B} 1=0.065 \\
\mathrm{~B} 2=0.053 \\
\mathrm{~B} 3=0.001\end{array}$ \\
\hline $3-$ & Chwirut1 & $\begin{array}{l}\mathrm{B} 1=0.1 \\
\mathrm{~B} 2=0.001 \\
\mathrm{~B} 3=0.002\end{array}$ & $\begin{array}{l}\mathrm{B} 1=0.19 \\
\mathrm{~B} 2=0.0061 \\
\mathrm{~B} 3=0.0105\end{array}$ & $\begin{array}{l}\mathrm{B} 1=0.157 \\
\mathrm{~B} 2=0.006 \\
\mathrm{~B} 3=0.012\end{array}$ & $\begin{array}{l}\mathrm{B} 1=0.089 \\
\mathrm{~B} 2=0.0229 \\
\mathrm{~B} 3=0.0002\end{array}$ \\
\hline 4- & Lanczos3 & $\begin{array}{l}\mathrm{B} 1=1.2 \\
\mathrm{~B} 2=0.3 \\
\mathrm{~B} 3=5.6 \\
\mathrm{~B} 4=5.5 \\
\mathrm{~B} 5=6.5 \\
\mathrm{~B} 6=7.6\end{array}$ & overflow & $\begin{array}{l}\mathrm{B} 1=2.447 \\
\mathrm{~B} 2=0.316 \\
\mathrm{~B} 3=648.2 \\
\mathrm{~B} 4=345.7 \\
\mathrm{~B} 5=648.2 \\
\mathrm{~B} 6=-2.79\end{array}$ & $\begin{array}{l}\text { B1 }=0.82 \\
\text { B2 }=0.11 \\
\text { B3 }=-1.55 \\
\text { B4 }=6.31 \\
\text { B5 }=3.24 \\
\text { B6 }=8.05\end{array}$ \\
\hline 5- & Gauss1 & $\begin{array}{l}\mathrm{B} 1=97 \\
\mathrm{~B} 2=0.009 \\
\mathrm{~B} 3=100 \\
\mathrm{~B} 4=65 \\
\mathrm{~B} 5=20 \\
\mathrm{~B} 6=70 \\
\mathrm{~B} 7=178 \\
\mathrm{~B} 8=16.5\end{array}$ & $\begin{array}{l}\mathrm{B} 1=98.77 \\
\mathrm{~B} 2=0.001 \\
\mathrm{~B} 3=100.48 \\
\mathrm{~B} 4=67.48 \\
\mathrm{~B} 5=23.12 \\
\mathrm{~B} 6=71.99 \\
\mathrm{~B} 7=178.9 \\
\mathrm{~B} 8=18.38\end{array}$ & $\begin{array}{l}\mathrm{B} 1=98.77 \\
\mathrm{~B} 2=0.01 \\
\mathrm{~B} 3=100.49 \\
\mathrm{~B} 4=67.48 \\
\mathrm{~B} 5=23.12 \\
\mathrm{~B} 6=71.99 \\
\mathrm{~B} 7=178.9 \\
\mathrm{~B} 8=18.38\end{array}$ & overflow \\
\hline $6-$ & Gauss2 & $\begin{array}{l}\mathrm{B} 1=96 \\
\mathrm{~B} 2=0.009 \\
\mathrm{~B} 3=103 \\
\mathrm{~B} 4=106 \\
\mathrm{~B} 5=18 \\
\mathrm{~B} 6=72 \\
\mathrm{~B} 7=151 \\
\mathrm{~B} 8=18\end{array}$ & $\begin{array}{l}\mathrm{B} 1=99 \\
\mathrm{~B} 2=0.010 \\
\mathrm{~B} 3=101.8 \\
\mathrm{~B} 4=107 \\
\mathrm{~B} 5=23.5 \\
\mathrm{~B} 6=72.04 \\
\mathrm{~B} 7=153.2 \\
\mathrm{~B} 8=19.5\end{array}$ & $\begin{array}{l}\mathrm{B} 1=99.1 \\
\mathrm{~B} 2=0.011 \\
\mathrm{~B} 3=101.87 \\
\mathrm{~B} 4=65 \\
\mathrm{~B} 5=23.57 \\
\mathrm{~B} 6=72.04 \\
\mathrm{~B} 7=153.27 \\
\mathrm{~B} 8=19.52\end{array}$ & overflow \\
\hline $7-$ & Danwood & $\begin{array}{l}\mathrm{B} 1=1 \\
\mathrm{~B} 2=5\end{array}$ & $\begin{array}{l}\mathrm{B} 1=0.768 \\
\mathrm{~B} 2=3.86\end{array}$ & $\begin{array}{l}\mathrm{B} 1=0.769 \\
\mathrm{~B} 2=3.86\end{array}$ & $\begin{array}{l}\mathrm{B} 1=0.768 \\
\mathrm{~B} 2=3.86\end{array}$ \\
\hline $8-$ & Misra1b & $\begin{array}{l}\mathrm{B} 1=500 \\
\mathrm{~B} 2=0.0001\end{array}$ & $\begin{array}{l}\mathrm{B} 1=337.98 \\
\mathrm{~B} 2=0.0003\end{array}$ & $\begin{array}{l}\mathrm{B} 1=332.84 \\
\mathrm{~B} 2=0\end{array}$ & $\begin{array}{l}\mathrm{B} 1=469.63 \\
\mathrm{~B} 2=0.0002\end{array}$ \\
\hline 9- & Kirby2 & $\begin{array}{l}\mathrm{B} 1=2 \\
\mathrm{~B} 2=0.1 \\
\mathrm{~B} 3=0.03 \\
\mathrm{~B} 4=-0.001 \\
\mathrm{~B} 5=0.00001\end{array}$ & $\begin{array}{l}\mathrm{B} 1=1.64 \\
\mathrm{~B} 2=0.13 \\
\mathrm{~B} 3=0.0025 \\
\mathrm{~B} 4=-0.0017 \\
\mathrm{~B} 5=0.000021\end{array}$ & $\begin{array}{l}\mathrm{B} 1=1.73 \\
\mathrm{~B} 2=0.14 \\
\mathrm{~B} 3=0.003 \\
\mathrm{~B} 4=-0.002 \\
\text { B5 }=0.000022\end{array}$ & $\begin{array}{l}\mathrm{B} 1=1.99 \\
\mathrm{~B} 2=0.19 \\
\mathrm{~B} 3=0.003 \\
\text { B4 }=-0.0002 \\
\text { B5 }=0.000024\end{array}$ \\
\hline $10-$ & Hahn1 & $\begin{array}{l}\mathrm{B} 1=10 \\
\mathrm{~B} 2=-1 \\
\mathrm{~B} 3=0.05 \\
\mathrm{~B} 4=-0.00001 \\
\mathrm{~B} 5=0.005 \\
\mathrm{~B} 6=0.001 \\
\mathrm{~B} 7=-0.1 . \mathrm{E}-5\end{array}$ & $\begin{array}{l}\mathrm{B} 1=3.14 \\
\text { B2 }=-0.44 \\
\text { B3 }=0.021 \\
\text { B4 }=-0.00037 \\
\text { B5 }=-0.026 \\
\text { B6 }=0.00154 \\
\text { B7 }=-1.62 . \mathrm{E}-5\end{array}$ & $\begin{array}{l}\mathrm{B} 1=1.07 \\
\mathrm{~B} 2=-0.122 \\
\mathrm{~B} 3=0.004 \\
\mathrm{~B} 4=-0.0000014 \\
\mathrm{~B} 5=-0.06 \\
\mathrm{~B} 6=0 \\
\mathrm{~B} 7=-1.22 . \mathrm{E}-7\end{array}$ & $\begin{array}{l}\mathrm{B} 1=11.63 \\
\mathrm{~B} 2=15.9 \\
\mathrm{~B} 3=26.69 \\
\mathrm{~B} 4=1.14 \\
\mathrm{~B} 5=58.1 \\
\mathrm{~B} 6=2.99 \\
\mathrm{~B} 7=0.05\end{array}$ \\
\hline & & $\begin{array}{l}\mathrm{B} 1=50 \\
\mathrm{~B} 2=150\end{array}$ & $\begin{array}{l}\mathrm{B} 1=10.36 \\
\mathrm{~B} 2=1016\end{array}$ & $\begin{array}{l}\mathrm{B} 1=10.36 \\
\mathrm{~B} 2=1279\end{array}$ & $\begin{array}{l}\mathrm{B} 1=10.35 \\
\mathrm{~B} 2=42.9\end{array}$ \\
\hline
\end{tabular}




\begin{tabular}{|c|c|c|c|c|c|}
\hline 11- & Mgh17 & $\begin{array}{l}\mathrm{B} 3=-100 \\
\mathrm{~B} 4=1 \\
\mathrm{~B} 5=1\end{array}$ & $\begin{array}{l}\mathrm{B} 3=-1016 \\
\mathrm{~B} 4=0.4 \\
\mathrm{~B} 5=0.39\end{array}$ & $\begin{array}{l}\mathrm{B} 3=-1280 \\
\mathrm{~B} 4=0.4 \\
\mathrm{~B} 5=0.39\end{array}$ & $\begin{array}{l}\mathrm{B} 3=-43.4 \\
\mathrm{~B} 4=0.46 \\
\mathrm{~B} 5=0.35\end{array}$ \\
\hline $\begin{array}{l}12- \\
13-\end{array}$ & Lanczos & $\begin{array}{l}\mathrm{B} 1=1.2 \\
\mathrm{~B} 2=0.3 \\
\mathrm{~B} 3=5.6 \\
\mathrm{~B} 4=5.5 \\
\mathrm{~B} 5=6.5 \\
\mathrm{~B} 6=7.6\end{array}$ & $\begin{array}{l}\mathrm{B} 1=1.2 \\
\mathrm{~B} 2=0.3 \\
\mathrm{~B} 3=5.6 \\
\mathrm{~B} 4=5.5 \\
\mathrm{~B} 5=6.5 \\
\mathrm{~B} 6=7.6\end{array}$ & $\begin{array}{l}\mathrm{B} 1=1.2 \\
\mathrm{~B} 2=0.3 \\
\mathrm{~B} 3=5.6 \\
\mathrm{~B} 4=5.5 \\
\mathrm{~B} 5=6.5 \\
\mathrm{~B} 6=7.6\end{array}$ & $\begin{array}{l}\mathrm{B} 1=1.2 \\
\mathrm{~B} 2=0.3 \\
\mathrm{~B} 3=5.6 \\
\mathrm{~B} 4=5.5 \\
\mathrm{~B} 5=6.5 \\
\mathrm{~B} 6=7.6\end{array}$ \\
\hline $14-$ & Gauss3 & $\begin{array}{l}\mathrm{B} 1=96 \\
\mathrm{~B} 2=0.0096 \\
\mathrm{~B} 3=80 \\
\mathrm{~B} 4=110 \\
\mathrm{~B} 5=25 \\
\mathrm{~B} 6=75 \\
\mathrm{~B} 7=139 \\
\mathrm{~B} 8=25\end{array}$ & $\begin{array}{l}\mathrm{B} 1=99.01 \\
\mathrm{~B} 2=0.109 \\
\mathrm{~B} 3=101.87 \\
\mathrm{~B} 4=107.03 \\
\mathrm{~B} 5=23.57 \\
\mathrm{~B} 6=72.57 \\
\mathrm{~B} 7=153.27 \\
\mathrm{~B} 8=19.53\end{array}$ & $\begin{array}{l}\mathrm{B} 1=99.01 \\
\mathrm{~B} 2=0.011 \\
\mathrm{~B} 3=101.88 \\
\mathrm{~B} 4=107.03 \\
\mathrm{~B} 5=23.57 \\
\mathrm{~B} 6=72.04 \\
\mathrm{~B} 7=153.27 \\
\mathrm{~B} 8=19.52\end{array}$ & $\begin{array}{l}\mathrm{B} 1=114.8 \\
\mathrm{~B} 2=0.0039 \\
\mathrm{~B} 3=33.96 \\
\mathrm{~B} 4=4127.43 \\
\mathrm{~B} 5=2245.5 \\
\mathrm{~B} 6=45.98 \\
\mathrm{~B} 7=437.7 \\
\mathrm{~B} 8=22.9\end{array}$ \\
\hline $15-$ & Misralc & $\begin{array}{l}\mathrm{B} 1=500 \\
\mathrm{~B} 2=0.001\end{array}$ & $\begin{array}{l}\mathrm{B} 1=636.39 \\
\mathrm{~B} 2=0.00002\end{array}$ & $\begin{array}{l}\mathrm{B} 1=626.04 \\
\mathrm{~B} 2=0\end{array}$ & $\begin{array}{l}\mathrm{B} 1=637.72 \\
\mathrm{~B} 2=0.0002\end{array}$ \\
\hline 16- & Misrald & $\begin{array}{l}\mathrm{B} 1=500 \\
\mathrm{~B} 2=0.001\end{array}$ & $\begin{array}{l}\mathrm{B} 1=437.34 \\
\mathrm{~B} 2=0.0003\end{array}$ & $\begin{array}{l}\mathrm{B} 1=437.47 \\
\mathrm{~B} 2=0\end{array}$ & $\begin{array}{l}\mathrm{B} 1=476.67 \\
\mathrm{~B} 2=0.00027\end{array}$ \\
\hline $17-$ & Roszman & $\begin{array}{l}\mathrm{B} 1=0.1 \\
\mathrm{~B} 2=\mathrm{E}-5 \\
\mathrm{~B} 3=1000 \\
\mathrm{~B} 4=-100\end{array}$ & $\begin{array}{l}\mathrm{B} 1=0.2 \\
\mathrm{~B} 2=6.18 . \mathrm{E}-6 \\
\mathrm{~B} 3=1204 \\
\mathrm{~B} 4=-180.82\end{array}$ & $\begin{array}{l}\mathrm{B} 1=0.202 \\
\mathrm{~B} 2=-6.184 . \mathrm{E}-6 \\
\mathrm{~B} 3=1205 \\
\mathrm{~B} 4=-180\end{array}$ & $\begin{array}{l}\mathrm{B} 1=0.249 \\
\mathrm{~B} 2=-1.35 . \mathrm{E}-5 \\
\mathrm{~B} 3=1000 \\
\mathrm{~B} 4=-100.0001\end{array}$ \\
\hline $18-$ & Enso & $\begin{array}{l}\mathrm{B} 1=11 \\
\mathrm{~B} 2=3 \\
\mathrm{~B} 3=0.5 \\
\mathrm{~B} 4=40 \\
\mathrm{~B} 5=-0.7 \\
\mathrm{~B} 6=-1.3 \\
\mathrm{~B} 7=25 \\
\mathrm{~B} 8=-0.3 \\
\mathrm{~B} 9=1.4\end{array}$ & $\begin{array}{l}\mathrm{B} 1=10.51 \\
\mathrm{~B} 2=3.08 \\
\mathrm{~B} 3=0.46 \\
\mathrm{~B} 4=44.17 \\
\mathrm{~B} 5=-1.63 \\
\mathrm{~B} 6=0.465 \\
\mathrm{~B} 7=26.84 \\
\mathrm{~B} 8=0.18 \\
\mathrm{~B} 9=1.5\end{array}$ & $\begin{array}{l}\mathrm{B} 1=10.512 \\
\mathrm{~B} 2=3.08 \\
\mathrm{~B} 3=0.461 \\
\mathrm{~B} 4=44.3 \\
\mathrm{~B} 5=1.61 \\
\mathrm{~B} 6=0.534 \\
\mathrm{~B} 7=26.88 \\
\mathrm{~B} 8=0.22 \\
\mathrm{~B} 9=1.497\end{array}$ & $\begin{array}{l}\mathrm{B} 1=10.51 \\
\mathrm{~B} 2=3.076 \\
\mathrm{~B} 3=0.532 \\
\mathrm{~B} 4=44.3 \\
\mathrm{~B} 5=1.61 \\
\mathrm{~B} 6=0.525 \\
\mathrm{~B} 7=26.887 \\
\mathrm{~B} 8=0.212 \\
\mathrm{~B} 9=1.496\end{array}$ \\
\hline 19- & Mgh09 & $\begin{array}{l}\mathrm{B} 1=25 \\
\mathrm{~B} 2=39 \\
\mathrm{~B} 3=41.5 \\
\mathrm{~B} 4=39\end{array}$ & $\begin{array}{l}\mathrm{B} 1=1.15 \\
\mathrm{~B} 2=2.53 \\
\mathrm{~B} 3=-2.51 \\
\mathrm{~B} 4=2.95\end{array}$ & $\begin{array}{l}\mathrm{B} 1=-0.002 \\
\mathrm{~B} 2=-3.88 \\
\mathrm{~B} 3=-2.71 \\
\mathrm{~B} 4=-18.69\end{array}$ & $\begin{array}{l}\mathrm{B} 1=3.91 \\
\mathrm{~B} 2=92.6 \\
\mathrm{~B} 3=116.3 \\
\mathrm{~B} 4=-18.69\end{array}$ \\
\hline $20-$ & Thurber & $\begin{array}{l}\mathrm{B} 1=1000 \\
\mathrm{~B} 2=1000 \\
\mathrm{~B} 3=400 \\
\mathrm{~B} 4=40 \\
\mathrm{~B} 5=0.7 \\
\mathrm{~B} 6=0.3 \\
\mathrm{~B} 7=0.03\end{array}$ & $\begin{array}{l}\mathrm{B} 1=1273.58 \\
\mathrm{~B} 2=1516.78 \\
\mathrm{~B} 3=369.9 \\
\mathrm{~B} 4=4.15 \\
\mathrm{~B} 5=0.87 \\
\mathrm{~B} 6=0.316 \\
\mathrm{~B} 7=0.102\end{array}$ & $\begin{array}{l}\mathrm{B} 1=1288 \\
\mathrm{~B} 2=1491 \\
\mathrm{~B} 3=583.23 \\
\mathrm{~B} 4=75.457 \\
\mathrm{~B} 5=0.966 \\
\mathrm{~B} 6=0.398 \\
\mathrm{~B} 7=-0.05\end{array}$ & $\begin{array}{l}\mathrm{B} 1=1287.49 \\
\mathrm{~B} 2=1472.12 \\
\mathrm{~B} 3=569.58 \\
\mathrm{~B} 4=72.78 \\
\mathrm{~B} 5=0.951 \\
\mathrm{~B} 6=0.39 \\
\mathrm{~B} 7=0.04\end{array}$ \\
\hline $21-$ & Boxbod & $\begin{array}{l}\mathrm{B} 1=100 \\
\mathrm{~B} 2=0.75\end{array}$ & $\begin{array}{l}\mathrm{B} 1=213.79 \\
\mathrm{~B} 2=0.547\end{array}$ & $\begin{array}{l}\mathrm{B} 1=213.81 \\
\mathrm{~B} 2=0.547\end{array}$ & $\begin{array}{l}\mathrm{B} 1=172.49 \\
\mathrm{~B} 2=0.687\end{array}$ \\
\hline $22-$ & Rat42 & $\begin{array}{l}\mathrm{B} 1=100 \\
\mathrm{~B} 2=1 \\
\mathrm{~B} 3=0.1\end{array}$ & $\begin{array}{l}\mathrm{B} 1=129 \\
\mathrm{~B} 2=2.437 \\
\mathrm{~B} 3=0.0406\end{array}$ & $\begin{array}{l}\mathrm{B} 1=129.305 \\
\mathrm{~B} 2=2.435 \\
\mathrm{~B} 3=0.04\end{array}$ & $\begin{array}{l}\mathrm{B} 1=72.46 \\
\mathrm{~B} 2=2.62 \\
\mathrm{~B} 3=0.067\end{array}$ \\
\hline $23-$ & Mgh10 & $\begin{array}{l}\mathrm{B} 1=2 \\
\mathrm{~B} 2=400000 \\
\mathrm{~B} 3=25000\end{array}$ & $\begin{array}{l}\mathrm{B} 1=5003.58 \\
\mathrm{~B} 2=312451 \\
\mathrm{~B} 3=32609.6\end{array}$ & $\begin{array}{l}\mathrm{B} 1=0.002 \\
\mathrm{~B} 2=-55220 \\
\mathrm{~B} 3=-7442\end{array}$ & $\begin{array}{l}\mathrm{B} 1=46276 . \mathrm{E}-7 \\
\mathrm{~B} 2=399999 \\
\mathrm{~B} 3=25000\end{array}$ \\
\hline $24-$ & Eckerle4 & $\begin{array}{l}\mathrm{B} 1=1 \\
\mathrm{~B} 2=10 \\
\mathrm{~B} 3=500\end{array}$ & $\begin{array}{l}\mathrm{B} 1=1.55 \\
\mathrm{~B} 2=4.089 \\
\mathrm{~B} 3=451.53\end{array}$ & $\begin{array}{l}\mathrm{B} 1=1.554 \\
\mathrm{~B} 2=4.089 \\
\mathrm{~B} 3=451.54\end{array}$ & $\begin{array}{l}\mathrm{B} 1=0.002 \\
\mathrm{~B} 2=10.06 \\
\mathrm{~B} 3=500.04\end{array}$ \\
\hline $25-$ & Rat43 & $\begin{array}{l}\mathrm{B} 1=700 \\
\mathrm{~B} 2=5 \\
\mathrm{~B} 3=0.75 \\
\mathrm{~B} 4=1.3\end{array}$ & $\begin{array}{l}\mathrm{B} 1=699.45 \\
\mathrm{~B} 2=5.33 \\
\mathrm{~B} 3=0.76 \\
\mathrm{~B} 4=1.297\end{array}$ & $\begin{array}{l}\mathrm{B} 1=698.98 \\
\mathrm{~B} 2=5.31 \\
\mathrm{~B} 3=0.76 \\
\mathrm{~B} 4=1.291\end{array}$ & $\begin{array}{l}\mathrm{B} 1=699.45 \\
\mathrm{~B} 2=5.33 \\
\mathrm{~B} 3=0.76 \\
\mathrm{~B} 4=1.3\end{array}$ \\
\hline $26-$ & Bennett5 & $\begin{array}{l}\mathrm{B} 1=-2000 \\
\mathrm{~B} 2=50 \\
\mathrm{~B} 3=0.8\end{array}$ & $\begin{array}{l}\mathrm{B} 1=-2114.5 \\
\mathrm{~B} 2=44.86 \\
\mathrm{~B} 3=0.963\end{array}$ & $\begin{array}{l}\mathrm{B} 1=-1341 \\
\mathrm{~B} 2=39.96 \\
\mathrm{~B} 3=1.057\end{array}$ & $\begin{array}{l}\mathrm{B} 1=-1999.4 \\
\mathrm{~B} 2=55.29 \\
\mathrm{~B} 3=0.978\end{array}$ \\
\hline $27-$ & Nelson & $\begin{array}{l}\mathrm{B} 1=2.5 \\
\mathrm{~B} 2=5 . \mathrm{E}-9 \\
\mathrm{~B} 3=-0.05\end{array}$ & $\begin{array}{l}\mathrm{B} 1=1.159 \\
\mathrm{~B} 2=2.8 . \mathrm{E}-6 \\
\mathrm{~B} 3=-0.03\end{array}$ & $\begin{array}{l}\mathrm{B} 1=2.638 \\
\mathrm{~B} 2=2.4 . \mathrm{E}-9 \\
\mathrm{~B} 3=-0.062\end{array}$ & $\begin{array}{l}\mathrm{B} 1=2.5 \\
\mathrm{~B} 2=2.8 . \mathrm{E}-8 \\
\mathrm{~B} 3=-0.05\end{array}$ \\
\hline
\end{tabular}




\subsection{Some Comments on Table.3}

1) Initial parameter estimates are taken from internet resource to make meaningful comparisons. Because in nonlinear modeling there are a lot of factors affecting to find global optimum. To decide which factor does really affect, some other factors should be taken constant.

2) In 13 of the total 26 cases, all packages produce similar estimates. Nevertheless, this is not a point that must be overemphasized. Because in nonlinear modeling it is possible to find totally different equations that fit exactly the same data.

3) In 4 of the situations out of 26 , the results of the three programs are totally different from each other probably due to different optimization algorithms followed and to different convergence criteria adopted.

4) Yet, to make a more meaningful comparison, some summarizing statistics on parameter estimators should have been found. This is a drawback for Excel-Solver(5) since it does not automatically generate an estimated variance-covariance matrix.

5) These drawbacks can be overcome by writing some macros in Visual Basic programming. One can refer to (3) and (4) for this purpose.

\section{Short discussion}

Although EXCEL Solver could not produce as many satisfactory models that seem fit well to data as the other packages, it is not too bad at all for all these 26 data sets. This is a result that has to be expected a priori. Because, the software programs like SPSS, and NCSS are designed to solve such complicated estimation problems. On the other hand Microsoft Excel Solver is a general purpose optimization tool. Perhaps this characteristic of Solver brings more flexibility and more freedom to scientists. Because manipulating and analysing data by a spreadsheet has its own advantages. The number of functions that can be written on any cell of an Excel file is almost infinite. Then optimizing an objective function defined on a target cell by adjusting some parameter estimates through Solver optimization is straightforward. To be more specific, as well as the least squares' and maximum likelihood functions, one can easily find optimum values of some objective functions defined arbitrarily and used in robust estimation.

Microsoft Excel provides its users a variety of facilities not only for computational issues but also for pedagogical matters especially for undergraduate statistics students. By its various ready-to-use macros and graphical presentations, Excel is a perfect companion especially for undergraduate statistics courses. Besides, by Microsoft Excel solver package, one can solve some optimization problems including nonlinear regression problems precisely .

\section{References}

[1] Bates, D.M., Watts, D.G., Nonlinear Regression Analysis and Its Applications, New York, John Wiley\&Sons, (1988).

[2] Bevington,P.R., Robinson,D.K., "Data Reduction and Error Analysis for the Physical Sciences”, McGraw Hill, Third edition, (2003) 148-151. 
[3] Billo, E.J., EXCEL for Scientists and Engineers Numerical Methods, Wiley- Interscience, John Wiley\&Sons, (2007).

[4] De Levie, R., Advanced Excel for Scientific Data Analysis, Oxford University Press, (2004).

[5] Huet,S., Bouvier,A., Gruet,M., Jolivet,E., Statistical Tools for Nonlinear Regression: A Practical Guide with S-Plus Examples, Springer-Verlag, New York, Springer Series in Statistics, (1996).

[6] Motulsky, H., Christopoulos, A., Fitting Models to Biological Data Using Linear and Nonlinear Regression: A Practical Guide to Curve Fitting, USA, Oxford University Press, (2004).

[7] Neter J., Wasserman W., Kutner M. H., "Applied Linear Statistical Models", Second edition,Illinois, Richard D. Irwin. (1985). 466-490.

[8] Ross, G.J.S., Nonlinear Estimation, Springer Series in Statistics , Springer-Verlag, (1990).

[9] Seber G.A.F., WILD C.J., "Nonlinear Regression”, USA, John Wiley\&Sons., (1989) 91102.

[10] http//itl.nist.gov/div898/strd/nls/nls_main.shtml 